\title{
FUNCTIONS WITH VALUES IN A GIVEN DOMAIN
}

W. K. HAYMAN

1. Suppose that $w=f(z)=a_{0}+a_{1} z+\cdots$ is regular in $|z|<1$ and takes there values lying in a domain $D$ in the $w$ plane. Let $A(R)$ be the radius of the largest circle whose centre lies on $|z|=R$, and whose interior lies in $D$.

In a recent paper Dvoretzky [2] has obtained some bounds for the coefficients of $f(z)^{\prime}$ when $f(z)$ is schlicht and restrictions are placed on the growth of $A(R)$ as a function of $R$. Suppose that

$$
A(R)=O\left(R^{\gamma}\right) .
$$

Then he has shown that

$$
\begin{array}{rr}
\left|a_{n}\right|=O\left(n^{\gamma /(2-\gamma)}\right), & 0<\gamma<1, \\
\left|a_{n}\right|=O\left(n^{-1 / 2+2 /(2-\gamma)}\right), & \gamma<-2,
\end{array}
$$

with intermediate result for intermediate values of $\gamma$.

We shall show (Theorem II) that the weaker hypothesis

$$
A(R)=o(R)
$$

is sufficient to imply

$$
\left|a_{n}\right|=O\left(n^{-1 / 2+e}\right)
$$

for every positive $\epsilon$ if $f(z)$ is schlicht, and

$$
\left|a_{n}\right|=O\left(n^{\varepsilon}\right)
$$

in the general case. The proof is a simple application of Schottky's theorem. We also obtain a result in the Bloch-Landau case when $A(R)$ is uniformly bounded (Theorem III).

2. Our results are based on the following theorem.

Theorem I. Suppose that with the notation of the previous section $\left|z_{0}\right|<1,\left|f\left(z_{0}\right)\right|=R$, where $A(R)<2 R / 3$. There exists an absolute constant $K$ such that we have

$$
\left|f^{\prime}\left(z_{0}\right)\right|<\frac{K A(R)}{1-\left|z_{0}\right|} .
$$

By integration of the inequality (2.1) we obtain bounds for the

Received by the editors June 1, 1951. 
maximum modulus of $f(z)$

$$
M[\rho, f]=\max _{|z|=\rho}|f(z)|,
$$

and hence using the Cauchy inequalities, or in the case of schlicht functions the area principle, bounds for the coefficients. These latter sharper bounds can also be obtained whenever the area principle holds, e.g., for finitely mean-valent functions.

We can deduce the following result.

Theorem II. Suppose that we have $A(R)<\epsilon R$ for $R>R_{0}$ where $\epsilon<2 / 3$. Then

$$
\begin{aligned}
M[\rho, f] & <\mu(1-\rho)^{-K_{\epsilon},} \\
\left|a_{n}\right| & <\mu e(n+1)^{K_{\epsilon}}, \quad n=1,2, \cdots .
\end{aligned}
$$

If further $f(z)$ is schlicht in $|z|<1$, then

$$
\left|a_{n}\right|<\mu e 2^{K_{\epsilon}} n^{-1 / 2+K_{\epsilon}}, \quad n=1,2, \cdots,
$$

where $\mu=\max \left(\left|a_{0}\right|, R_{0}\right)$. In particular if the hypothesis holds for every positive $\epsilon$, so does the conclusion.

We also consider the case when $A(R)$ is uniformly bounded. In this case Dvoretzky [2] obtained

$$
\left|a_{n}\right|=O\{\log n\}
$$

for a schlicht function $f(z)$. We can use the bound $L \geqq 1 / 2$ obtained by Ahlfors [1] for the Bloch-Landau constant to prove the following theorem.

Theorem III. Suppose that $A(R) \leqq C, 0 \leqq R<\infty$, where $C$ is a constant. Then we have

$$
\begin{aligned}
M\left[\rho, f(z)-a_{0}\right] & \leqq C \log \frac{1+\rho}{1-\rho}, \\
\left|a_{n}\right| & <e C, \quad n=1,2, \cdots .
\end{aligned}
$$

If further $f(z)$ is schlicht, we have given $\epsilon, 0<\epsilon<1$,

$$
\left|a_{n}\right|<C(1+\epsilon) n^{-(1 / 2)} \log (6 n / \epsilon), \quad n=1,2, \cdots .
$$

3. Proofs. We proceed to prove Theorem I. Let $f\left(z_{0}\right)=w_{0}$ where $\left|w_{0}\right|=R$. Let $w_{1}$ be a point on the circle $|w|=R$ such that

$$
\left|w_{1}-w_{0}\right|=3 A(R) \text {. }
$$

Since by hypothesis $3 A(R)<2 R, w_{1}$ exists. Now choose $a, b$ not in $D$ 
such that

$$
\left|a-w_{0}\right| \leqq A(R), \quad\left|b-w_{1}\right| \leqq A(R) .
$$

We put $\rho=1-\left|z_{0}\right|$, and consider

$$
g(z)=\frac{f\left(z_{0}+\rho z\right)-a}{b-a} .
$$

Then $g(z)$ is regular and $g(z) \neq 0$ or 1 in $|z|<1$. Also

$$
|b-a| \geqq\left|w_{1}-w_{0}\right|-\left|a-w_{0}\right|-\left|b-w_{1}\right| \geqq A(R),
$$

so that

$$
|g(0)|=\left|\frac{a-w_{0}}{b-a}\right| \leqq \frac{A(R)}{A(R)}=1 .
$$

Thus we have by Schottky's theorem $\left|g^{\prime}(0)\right| \leqq K / 5$, where $K$ is an absolute constant, and since $|b-a| \leqq\left|w_{1}-w_{0}\right|+\left|w_{1}-b\right|+\left|w_{0}-a\right|$ $\leqq 5 A(R)$, we deduce

$$
\rho\left|f^{\prime}\left(z_{0}\right)\right|=|b-a|\left|g^{\prime}(0)\right| \leqq 5 A(R) K / 5=K A(R) .
$$

This proves Theorem $I$.

We proceed to deduce Theorem II. Suppose that with the hypotheses of that theorem

$$
\left|f\left(\rho e^{i \theta}\right)\right|=M[\rho, f]>R_{0} .
$$

Let $r$ be the smallest non-negative number such that $\left|f\left(t e^{i \theta}\right)\right|>R_{0}$, $r<t<\rho$. Then we have clearly $\left|f\left(r e^{i \theta}\right)\right| \leqq \mu$. We put $R=\left|f\left(t e^{i \theta}\right)\right|$ and have by (2.1)

$$
\frac{d R}{d t} \leqq \frac{K \epsilon R}{1-t}, \quad r<t<\rho .
$$

This yields on integration

$$
\log \frac{R(\rho)}{\mu} \leqq K \epsilon \log \frac{1-r}{1-\rho} \leqq K \epsilon \log \frac{1}{1-\rho},
$$

which proves (2.3). Next we take $\rho=1-1 /(n+1)=n /(n+1)$ and deduce

$$
\begin{aligned}
\left|a_{n}\right| & \leqq \frac{M[\rho, f]}{\rho^{n}} \leqq\left(1+\frac{1}{n}\right)^{n} \mu(n+1)^{K_{\epsilon}}<e \mu(n+1)^{K_{\epsilon}} \\
& \leqq \mu e 2^{K_{\bullet} n^{K_{\epsilon}}},
\end{aligned}
$$


which gives (2.4). If further $f(z)$ is schlicht, the area of the image of the circle $|z|<\rho$ by $f(z)$ is at most $\pi\{M[\rho, f]\}^{2}$, so that

$$
\sum_{n=1}^{\infty} n\left|a_{n}\right|^{2} \rho^{2 n} \leqq[M[\rho, f]]^{2} .
$$

Thus we may replace $\left|a_{n}\right|$ by $n^{1 / 2}\left|a_{n}\right|$ in (3.2) so that (2.5) also follows. This completes the proof of Theorem II.

Ahlfors [1] has shown that the Bloch-Landau constant $L$ satisfies $L \geqq 1 / 2$. Thus if $f(z)=z+\cdots$ is regular in $|z|<1, f(z)$ takes all values in a circle of radius at least $1 / 2$. To prove Theorem III we apply this result to

$$
g(z)=\left\{f\left(\frac{z_{0}+z}{1+\bar{z}_{0} z}\right)-f\left(z_{0}\right)\right\} /\left[\left(1-\left|z_{0}\right|^{2}\right) f^{\prime}\left(z_{0}\right)\right]=z+\cdots
$$

which is regular in $|z|<1$ and omits values in every circle of radius greater than $C /\left[\left(1-\left|z_{0}\right|^{2}\right)\left|f^{\prime}\left(z_{0}\right)\right|\right]$ by hypothesis. Thus

$$
\begin{gathered}
C /\left[\left(1-\left|z_{0}\right|^{2}\right)\left|f^{\prime}\left(z_{0}\right)\right|\right] \geqq \frac{1}{2}, \\
\left|f^{\prime}\left(z_{0}\right)\right| \leqq \frac{2 C}{1-\left|z_{0}\right|^{2}}
\end{gathered}
$$

We apply Cauchy's inequality to $f^{\prime}(z)=\sum_{n=1}^{\infty} n a_{n} z^{n-1}$, and deduce

$$
n\left|a_{n}\right| \leqq \frac{1}{\rho^{n-1}} M\left[\rho, f^{\prime}\right] \leqq \frac{2 C}{\rho^{n-1}\left(1-\rho^{2}\right)} .
$$

If we put $\rho^{2}=(n-1) /(n+1)$, this gives

$$
\left|a_{n}\right| \leqq C \frac{n+1}{n}\left(\frac{n+1}{n-1}\right)^{(n-1) / 2}=C\left(\frac{(n+1)^{n+1}}{n^{2}(n-1)^{n-1}}\right)^{1 / 2}<e C .
$$

This proves (2.7) for $n \geqq 2$. For $n=1$ the result follows from (3.3).

Next we have, using (3.3),

$$
\left|f\left(\rho e^{i \theta}\right)-a_{0}\right| \leqq \int_{0}^{\rho}\left|f^{\prime}\left(t e^{i \theta}\right)\right| d t \leqq 2 C \int_{0}^{\rho} \frac{d t}{1-t^{2}}=C \log \frac{1+\rho}{1-\rho},
$$

which proves (2.6). Lastly if $f(z)$ is schlicht, we have from the area principle

$$
n^{-1 / 2}\left|a_{n}\right| \leqq \frac{M\left[\rho, f-a_{0}\right]}{\rho^{n}} \leqq \frac{C}{\rho^{n}} \log \frac{1+\rho}{1-\rho},
$$


and, if we put $\rho=(1+\epsilon)^{-1 / n}$, where $0<\epsilon<1$, this gives

$$
\left|a_{n}\right| \leqq C n^{-1 / 2}(1+\epsilon) \log \frac{(1+\epsilon)^{1 / n}+1}{(1+\epsilon)^{1 / n}-1}<C(1+\epsilon) n^{-1 / 2} \log \frac{6 n}{\epsilon},
$$

which is (2.8).

4. The functions $((1+z) /(1-z))^{2 e / \pi}$ and $(2 C / \pi) \log ((1+z) /(1-z))$ show that the orders of magnitude obtained in (2.3) and (2.6) are fairly sharp. Also in (2.7) $e C$ cannot be replaced by any quantity less than $C$ as the function $C z^{n}$ shows for each value of $n$. It is possible however that under sufficiently strong conditions one can show that $a_{n} \rightarrow 0$. This is the strongest result that is true for bounded general functions. One may ask also whether the orders of magnitude in (2.4), (2.5), and (2.8) can be improved.

In connection with Theorem III we note that (2.7) is an immediate consequence of (3.3). Thus (2.7) holds more generally if $f(z)$ belongs to a class of functions $f(z)$, which contains with $f(z)$ all functions of the form $f\left(\left(z_{0}+z\right) /\left(1+\bar{z}_{0} z\right)\right)$ for $\left|z_{0}\right|<1$, and such that $\left|f^{\prime}(0)\right| \leqq 2 C$ for every member of the class. This is true for instance if $f(z)$ maps no subdomain of the unit circle onto a schlicht circle of radius greater than $2 B C$, where $B$ is Bloch's constant, so that in this case (2.7) again holds. Thus "Bloch functions" also have uniformly bounded coeffcients, a fact which has not been previously noticed, I believe.

\section{REFERENCES}

1. L. V. Ahlfors, An extension of Schwarz's lemma, Trans. Amer. Math. Soc. vol. 43 (1938) pp. 359-364.

2. A. Dvoretzky, Bounds for the coefficients of univalent functions, Proceedings of the American Mathematical Society vol. 1 (1950) pp. 629-635.

University College, Exeter 